\title{
CONSERVATION DU SPERME DE VERRAT SANS DILUTION : CONDITIONS D'EXAMEN
}

PAR

\section{F. DU MESNIL DU BUISSON ( $\left.{ }^{1}\right)$}

Station de Recherches de Physiologie animale, Centre national de Recherches zootechniques, Jouy-en-Josas.

\section{INTRODUCTION}

Alors que le sperme de Verrat conservé en présence de liquides biologiques (jaune d'œuf ou lait) se prête à un examen analogue à celui qui permet d'apprécier la vitalité du sperme de Bélier ou de Taureau (LAsley et Phillips, I939; Roy, I955 ; Polge, I956 ; DU Mesnil. DU BUISSON, I956) le sperme conservé pur tombe très rapidement dans un état de mort apparente que les conditions habituelles de réchauffement entre lame et lamelle sont incapables de faire cesser. Dès 1933, MrıovaNov et HABIBULIN mentionnaient pour le sperme de Lapin et d'Etalon cette léthargie des spermatozoïdes sous le nom d'anabiose.

Alors que ces derniers auteuts attribuaient l'état d'anabiose à 1'alcalinité des sécrétions accessoires, Itro, Niwa, Kudo et Mizuho (I948) constatèrent que l'inanition des spermatozoïdes intervient aussi rapidement après sédimentation des spermatozoïdes qu'après dilution au tiers dans le chlorure de sodium isotonique, bien que dans ces deux cas les gamètes se trouvent à un $\mathrm{pH}$ inférieur à celui des sécrétions accessoires, et ils montrèrent que 1'agitation prolongée des échantillons permettait au sperme de recouvrer sa motilité. De plusieurs essais ils conclurent que les conditions les meilleures seraient réalisées par un séjour de I à 2 heures dans la poche. Noli (I950) comme POLGE (I956) parlent également d'une agitation de 2 heures pour l'examen du sperme.

L'ignorance de cette anabiose, ou la mauvaise utilisation de la méthode d'agitation avant examen, a conduit à un certain nombre d'erreurs et d'imprécisions (MC Kenzie, Miller et BaUguess, I938; IEWIS, I9I ; NOLL, I950) dans le diagnostic de la valeur des échantillons conservés.

(1) Avec la collaboration technique de J. Goussopoulos. 
Ainsi se pose, pour l'examen du sperme pur, le choix d'une méthode standardisée d'examen. Dans le travail que nous rapportons, nous avons cherché à définir les conditions les meilleures de reviviscence. Puis en les utilisant, nous avons comparé les effets de différents facteurs sur la conservation même du sperme de Verrat non dilué.

\section{MATÉRIEL ET MÉTHODES}

Nous avons employé 40 éjaculats non fractionnés d'une concentration moyenne de 258.000 spermatozoides $/ \mathrm{mm}^{3}$, provenant de 5 verrats âgés de 4 à 5 ans. La partie solide du sperme a toujours été écartée.

I'agitation du sperme a été réalisée de la façon suivante : des tubes de I cm de diamètre contenant I,5 cc de sperme sont posés dans des porte-tubes métalliques, eux-mêmes placés dans des cuves à fond plat remplies d'eau à température constante. Un mouvement de va-et-vient est assuré par l'intermédiaire d'un système bielle-manivelle actionné par un petit moteur électrique.

Les échantillons ont été examinés au microscope à contraste de phase, sans chauffage de la platine. On a compté le pourcentage de spermatozoïdes motiles et noté la motilité moyenne de o à 5. Les courbes rendant compte de l'état du sperme au cours de la conservation ont été établies en prenant comme valeur de l'échantillon le produit du pourcentage de spermatozoïdes motiles par la note de motilité.

\section{RÉSULTATS}

\section{I. - Conditions d'examen}

On a utilisé du sperme conservé pur 24 heures à la température ambiante $\left(\mathrm{I} 7^{\circ}\right.$ à $\left.20^{\circ} \mathrm{C}\right)$ et à $1^{\prime}$ abri de la lumière.

\section{A. - Mode et Durée d'agitation.}

Les échantillons étant plongés dans une cuve maintenue pendant l'agitation à $38^{\circ}$, trois sortes de mouvement ont été essayés dont les caractéristiques sont les suivantes :

\begin{tabular}{|c|c|c|c|}
\hline & Amplitude & $\begin{array}{l}\text { Fréquence } \\
\text { AR/minute }\end{array}$ & $\begin{array}{l}\text { Vitesse linéaire } \\
\text { moyenne en mètre/s. }\end{array}$ \\
\hline$V_{I} \ldots \ldots \ldots \ldots$ & 6 & II 5 & $0,23 \mathrm{~m} / \mathrm{s}$ \\
\hline$V_{2} \ldots \ldots \ldots \ldots$ & 4,5 & 200 & $0,30 \mathrm{~m} / \mathrm{s}$ \\
\hline$V_{3} \ldots \ldots \ldots \ldots$ & 6 & 200 & $0,40 \mathrm{~m} / \mathrm{s}$ \\
\hline Vo $\ldots \ldots \ldots \ldots$ & aucun & vement & \\
\hline
\end{tabular}

De 1'examen des courbes de la figure I on feut tirer plusieurs conclusions :

I ${ }^{0}$ Dans les différentes conditions où nous nous sommes placés, 
il est superflu et même nuisible de prolonger l'agitation pendant 2 heures, car au bout d'une demi-heure le sperme a déjà retrouvé le maximum de ses qualités. Ce temps de 2 heures retenu par Nol, et par PolgE d'après les conclusions des auteurs japonais ne correspond pas du reste exactement à la valeur rapportée dans le texte japonais lui-même pour la durée utile d'agitation puisque avec la méthode " de la poche " on relève un palier à partir de I heure et jusqu'à 2 heures avec 80 à $85 \mathrm{p}$. Ioo de spermatozoïdes motiles.

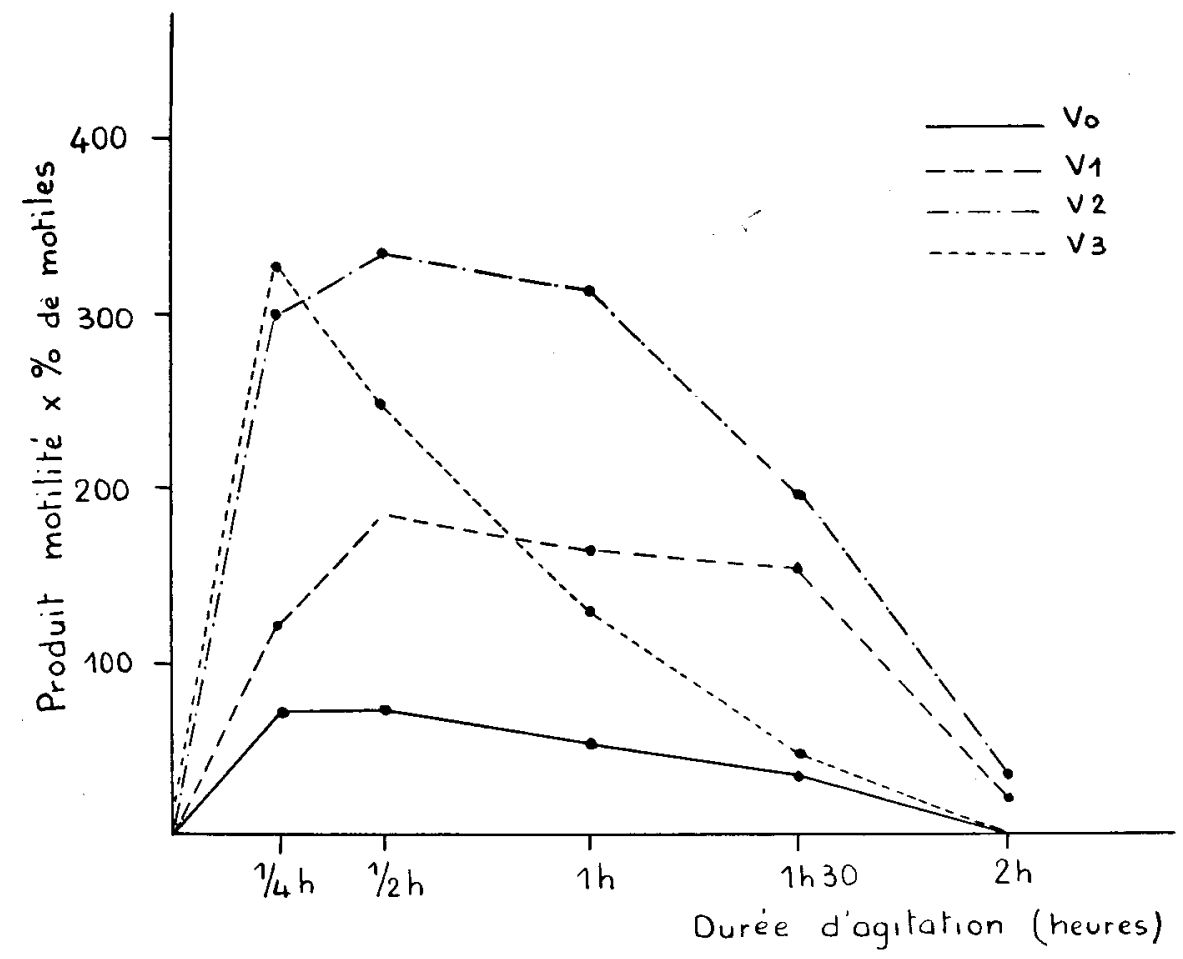

FIG. I.

$2^{\circ}$ L'élévation de température seule est incapable de ranimer le sperme, mais l'agitation, même modérée (VI) améliore considérablement la motilité des spermatozoïdes et le nombre de motiles.

$3^{\circ}$ A la vitesse $V_{3}$ la motilité maximum est atteinte plus vite, mais diminue rapidement. La vitesse $V_{2}$ a sur $V_{3}$ l'avantage de laisser le sperme à sa valeur la meilleure pendant une demi-heure, ce qui permet l'examen simultané de nombreux échantillons.

\section{B. - Mode de rêchauffement.}

Craignant que le réchauffement brutal du sperme depuis sa température de conservation jusqu'à $38^{\circ}$ ne soit en partie cause de la chute que 
l'on constate après une heure de traitement, nous avons commencé par placer nos échantillons dans l'eau à la température ambiante avant de les agiter. Les échantillons ont été ensuite secoués à la vitesse V2, en même temps que la température de l'eau et la leur s'élevaient lentement (40 minutes) de $20^{\circ} \mathrm{C}$ environ à $3^{\circ}$.

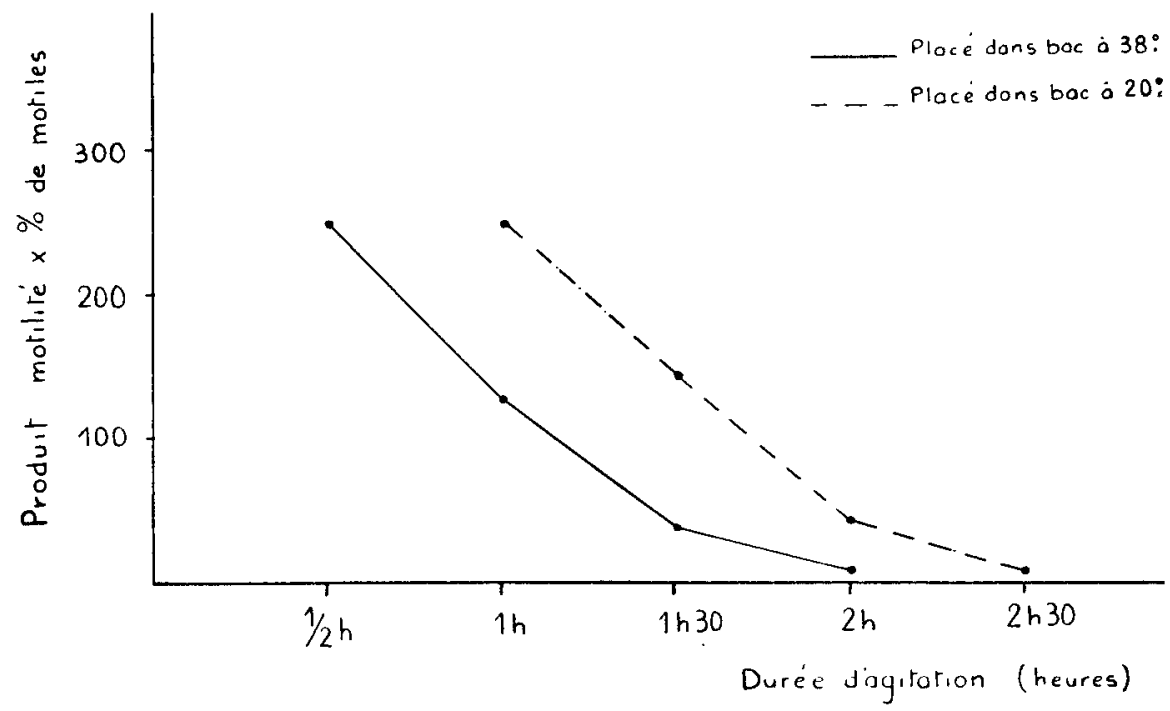

Fig. 2.

Nous n'avons obtenu aucune amélioration par cette méthode, mais seulement une translation des courbes (fig. 2) : la période où le sperme retrouve ses qualités se place une demi-heure plus tard, mais sa durée est du même ordre que précédemment (maximum 2 heures).

\section{C. - Atmosphère gazeuse au contact du sperme.}

Nous avons téalisé 4 lots de tubes:

I. - témoins ;

2. - tubes hermétiquement bouchés en remplaçant l'air par de l'oxygène ;

3. - tubes hermétiquement bouchés après remplacement de 1'air par du gaz carbonique;

4. - tubes hermétiquement bouchés après avoir fait le vide (pression de I à $2 \mathrm{~mm}$ de mercure).

Après une demi-heure à une heure d'agitation sous vide ou sous gaz carbonique, on ne constate absolument aucune reviviscence.

L'agitation en atmosphère d'oxygène non renouvelé donne des résultats sensiblement inférieurs à l'agitation en présence d'air. L'introduction d'air après agitation pendant une demi-heure ou une heure sous 
oxygène ne permet pas au sperme de retrouver des qualités comparables au témoin.

Une période d'agitation sots vide, avant agitation en présence d'air, est tout à fait nuisible ; la plupart du temps les spermatozoïdes sont tués par une heure d'agitation sous vide.

Au contraire, l'agitation préalable sous gaz carbonique pendant une demi-heure ou même une heure n'empêche pas le sperme de retrouver ensuitesa vitalité maximum par une agitation sous une atmosphère normale.

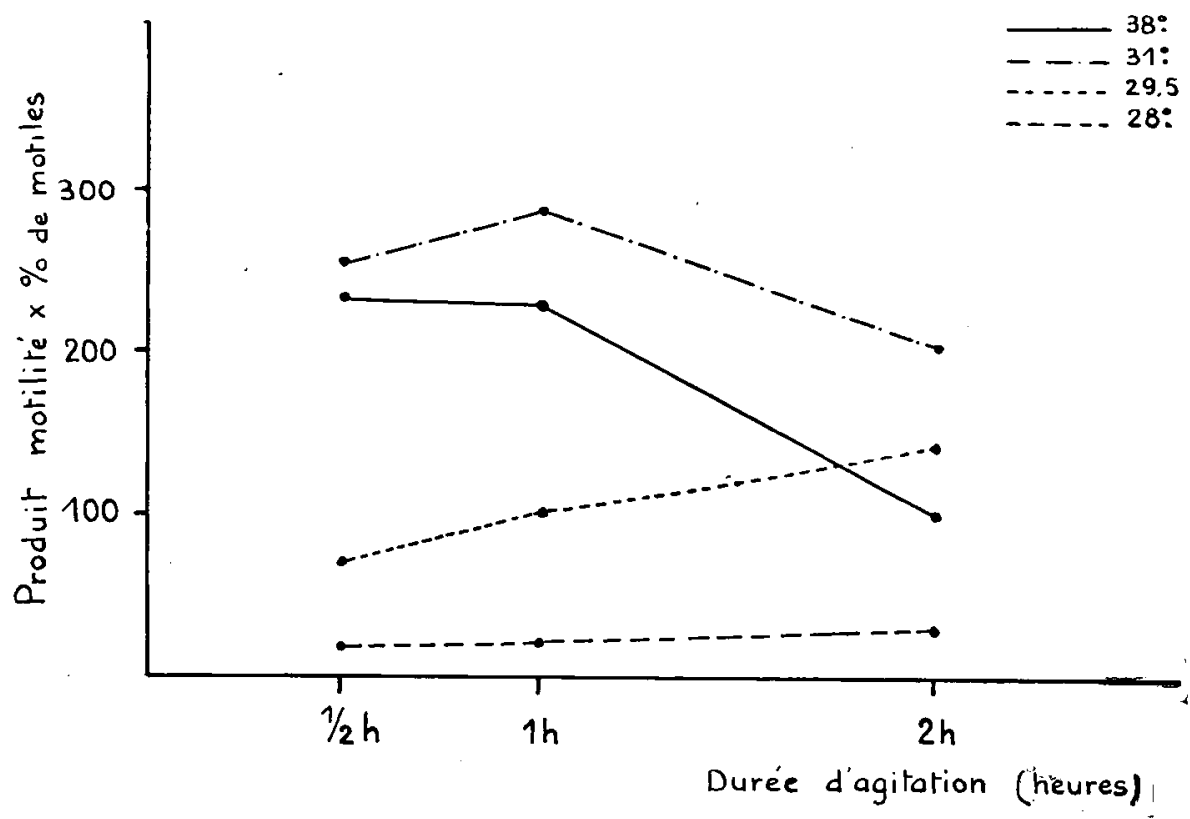

FIG. 3.

Il est donc établi que parmi nos 4 lots, seuls les échantillons agités en atmosphère oxygénée sont capables de reviviscence. Ces résultats rejoignent ceux de PoLGe qui note que les échantillons gardés sous azote ne peuvent être réactivés avant que l'on ait réintroduit de l'air. Du reste, en faisant barboter de l'oxygène bulle à bulle dans du sperme non agité et chauffé à $38^{\circ}$, on obtient également une bonne reviviscence ; nous avons cependant abandonné cette dernière méthode car il est difficile d'obtenir d'une façon simple par barbotage, des conditions " standard " d'un échantillon à l'autre. De plus, il se forme de la mousse qui gêne l'examen.

\section{D. - Température d'agitation.}

La vitesse d'agitation la meilleure, V2, ayant été employée, nous avons cherché à déterminer la température minimum qui permette d'obtenir la reviviscence du sperme agité (voir figure 3). 
L'agitation à $28^{\circ} \mathrm{C}$ ne permet qu'une reviviscence extrêmement faible du sperme, au contraire un réchauffement à $3 \mathrm{I}^{\circ}$ est suffisant.

La nécessité de l'élévation de température ressort également des essais des auteurs japonais. Avec des fréquences de mouvement comparables à celles que nous avons utilisées ( 80 à I60 par minute), ils n'obtiennent que des résultats faibles en ne réalisant pas simultanément le chauffage et l'agitation.

Après agitation à $29^{\circ} 5$, température voisine de celle de la poche d'après ITo, Niwa, Kudo et Mizuno (I948), certains des échantillons se sont montrés incapables de reprendre vie, tandis que d'autres finissaient par égaler les échantillons à $3^{\circ}{ }^{\circ} \mathrm{C}$. Il semble donc que l'agitation à cette température puisse être utilisée pour différencier les éjaculats. Quoiqu'il en soit, les résultats à $29^{\circ} 5 \mathrm{C}$ sont en moyenne sensiblement inférieurs à ceux que $1^{\prime}$ on obtient à partir de $31^{\circ} \mathrm{C}$.

Les deux éléments, oxygénation profonde réalisée par une bonne agitation et élévation de température, sont donc également nécessaires. Ainsi les conditions les meilleures pour l'examen du sperme sont les suivantes:

I $/ 2$ heure à I heure d'agitation (vitesse V2) des échantillons maintenus à une température comprise entre $31^{\circ} \mathrm{C}$ et $3^{\circ} \mathrm{C}$.

\section{II. - Conservation du sperme non dilué}

Des résultats contradictoires ont été publiés par certains auteurs au sujet de la conservation du sperme de Verrat non dilué. Les uns comme Mc Kenzie, Miller et Bauguess (I938) ont noté le temps au bout duquel aucun spermatozoïde ne bouge (durée de survie de 2 à I4 jours à $1 \mathrm{O}^{\circ}-\mathrm{I} 2^{\circ} \mathrm{C}$, variant en fonction de la fréquence des collectes), alors que d'autres comme LEWIS, considèrent au contraire, le sperme comme mort en $\mathrm{I} 4$ heures à $30^{\circ} \mathrm{C}$, en 24 heures à $\mathrm{I} 5$ ou $20^{\circ} \mathrm{C}$. Ce dernier auteur note également une action mortelle de la lumière en 2 heures. Le défaut d'oxygénation au moment de l'examen explique ces erreurs et ces imprécisions.

En examinant le sperme après agitation, ITo, Niwa, Kudo et Mrzuno (I948) montrent qu'il peut retrouver sa motilité après 5 jours de conservation à $15^{\circ}-20^{\circ} \mathrm{C}$. D'après NiwA, Mizuho et IsHikawa (I952) l'addition de sulfamides augmente la durée de la survie.

Les résultats de POLGE, qui prend des précautions d'examen équivalentes, lui permettent d'insister sur les différences de conservation entre le sperme éjaculé entier ou fractionné : à une température égale ou supérieure à $15^{\circ} \mathrm{C}$, la durée de conservation est similaire (sperme bon pendant 2 à 3 jours), au contraire, à $5^{\circ} \mathrm{C}$, le sperme entier est très mauvais après 24 heures ( 5 à Io \% de vivants) alors que l'utilisation de la fraction riche permet la conservation d'une très bonne motilité durant 8 à Io jours. 
Les résultats que nous publions ici ont été obtenus uniquement avec le sperme total conservé à la température ambiante $\left(\mathrm{I} 7-20^{\circ} \mathrm{C}\right)$ et à l'abri de la lumière. Les conditions d'examen ont été décrites précédemment.

I,e lendemain et le surlendemain de la collecte, 70 p. Ioo des spermatozoïdes sont vivants avec une bonne motilité $(3,5)$. Le $3^{\text {e jour et les }}$ suivants, la motilité, puis le pourcentage de vivants, s'effondrent. Dans tous les cas, nous nous sommes limités pour l'examen, au $5^{\mathrm{e}}$ jour de conservation.

Nous avons cherché à améliorer ce résultat :

- par addition de sulfamides pour empêcher les développements bactériens et éventuellement abaisser le métabolismeet favoriser 1'anabiose;

- par modification de l'atmosphère au-dessus du sperme durant la conservation, en vue de hâter ou d'approfondir l'anabiose.

\section{A. - Addition de sulfamides.}

Nous référant aux résultats de Niwa, Mizuho et Ishikawa (1952) nous avons cherché à utiliser comme eux la sulfamérazine sodique et l'homosulfamine. Ayant à notre disposition 4 spécialités pharmaceutiques du commerce, nous les avons employées isolément, puis en mélange, en reconstituant les doses indiquées par les auteurs japonais (fig. 4).

La sulfamérazine $(Q$ et $\mathrm{L})$ seule $(2 \mathrm{mg} / \mathrm{cc})$ donne des résultats légèrement inférieurs au témoin $(\mathrm{T})$.

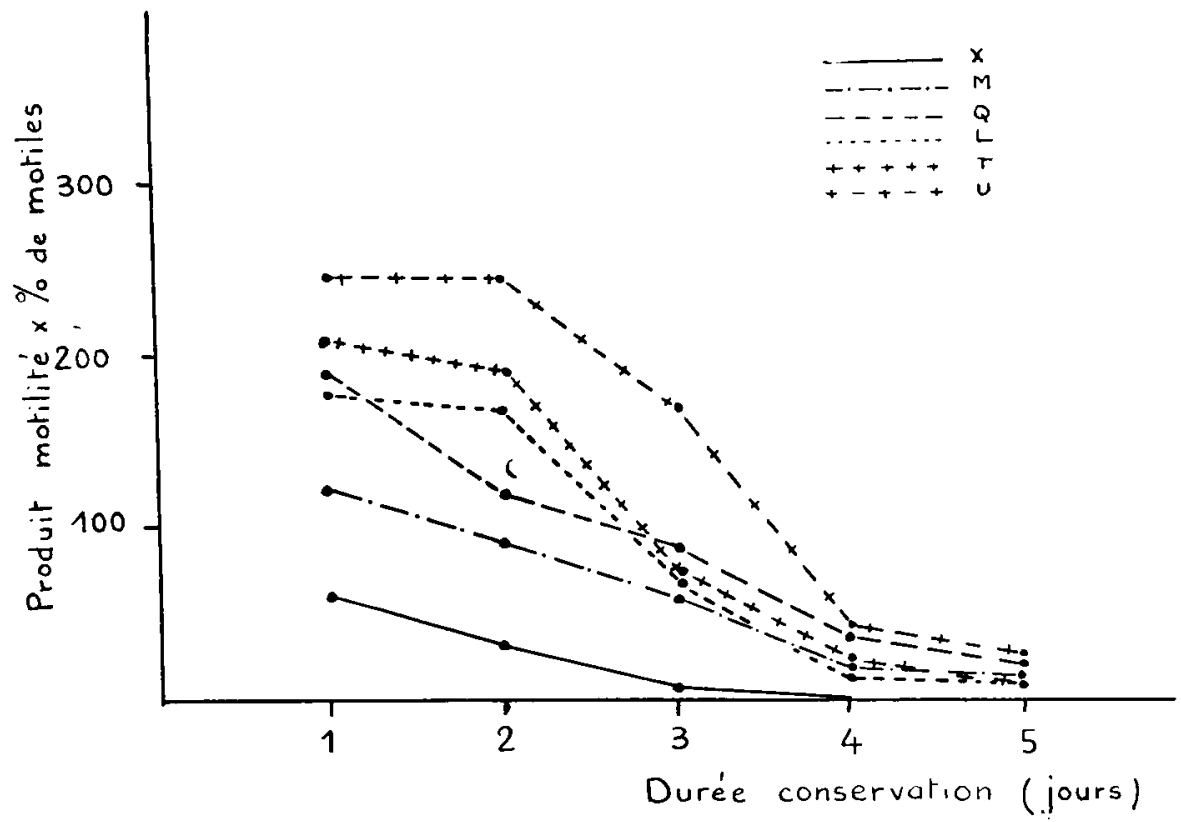

Fig. 4. 
L'homosulfamide (M) seule (I $\mathrm{mg} / \mathrm{cc}$ ) s'est montrée inférieure au témoin.

Le mélange des deux produits $(\mathrm{X})$ aux mêmes doses que précédemment est très nuisible au sperme de même que les combinaisons faisant rentrer une grosse quantité $(9 \mathrm{mg} / \mathrm{cc}$ ) de 4-aminophényl-sulfonamide.

Inversement, ce dernier produit, utilisé en mélange avec 1'homosulfamine, en quantité faible (courbe U) $(0,3 \mathrm{mg}$ d'homosulfamine et $2,7 \mathrm{mg}$ de 4-aminophényl-sulfonamide pour I cc) permet une nette amélioration de la conservation du sperme (notamment le $3^{\mathrm{e}}$ jour).

\section{B. - Modification de l'atmosphère gazeuse au contact du sperme durant la conservation.}

La présence d'oxygène ou son absence (vide, couche d'huile de paraffine au-dessus de l'échantillon) ne semblent guère modifier les conditions de conservation (fig. 5). Ceci rejoint les conclusions de Nol,L, à savoir que 1'aérobiose ou l'anaérobiose ne changent rien à la conservation du sperme.

Au contraire, la substitution d'une atmosphère de gaz carbonique à l'air durant la conservation permet une reviviscence du sperme très nettement supérieure jusqu'au $4^{\mathrm{e}}$ jour. Nous avons constaté avec le gaz carbonique une très grande régularité dans la conservation de la vitalité après 3 jours $(6$ échantillons sur 8 furent notés: 70 p. roo de vivants et 3,5 de motilité le $3^{\mathrm{e}}$ jour). Cet effet favorable ne persiste pas

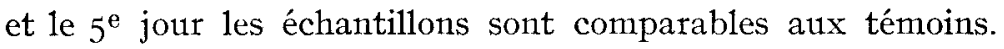

La nette supériorité des solutions contenant du bicarbonate de sodium (DU Mesnil du Buisson, I956) sur celles qui en sont dépourvues, pour la conservation du sperme de Verrat en présence de jaune d'œuf, peut faire penser que là également, l'amélioration est due à la libération, au sein même du dilueur, de gaz carbonique issu du bicarbonate.

Ainsi, de toutes façons, la durée de survie que nous obtenons pour le sperme sans dilution est inférieure à celle qui a été observée par les auteurs japonais dans ces conditions, et par d'autres auteurs et nousmêmes après dilution en présence de jaune d'œuf ou de lait.

Mais la pratique de l'insémination artificielle bovine montre que c'est d'une très bonne conservation pendant 2 jours que dépend son succès, et non d'une conservation médiocre même très prolongée, et les bons résultats obtenus dans la conservation du pouvoir fécondant avec le sperme conservé non dilué, en même temps que les résultats décevants d'insémination artificielle obtenus avec le sperme conservé plus de 24 heures en présence de jaune d'œuf, incitent à utiliser la technique de conservation sans dilution. 


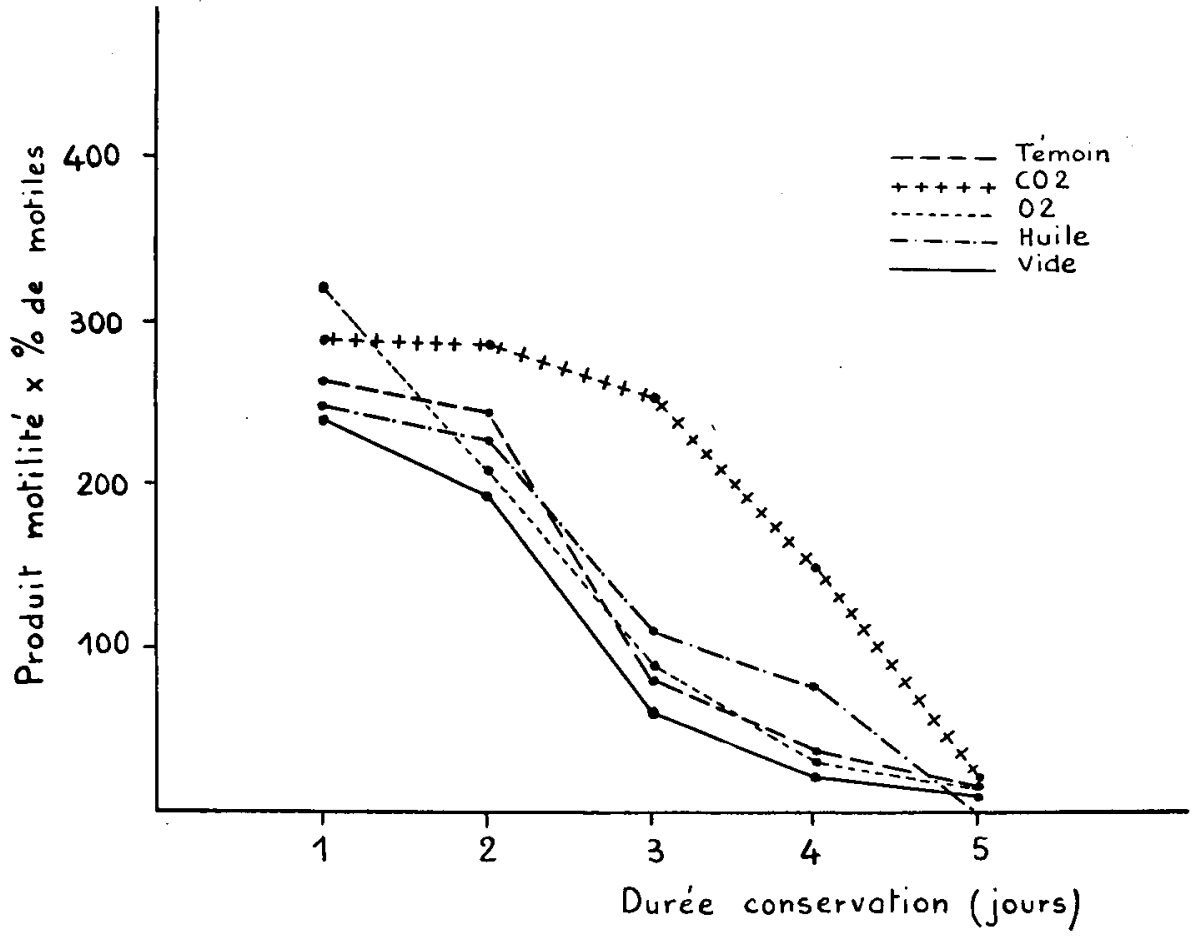

Fig. 5 .

C'est pourquoi nous suggérons que le sperme soit conservé pur, sous une atmosphère de gaz carbonique et nous proposons les conditions suivantes pour son examen: Agitation à une vitesse linéaire moyenne de $0,3 \mathrm{~m} /$ seconde par un mouvement alternatif de fréquence $200 /$ minute, pendant $I / 2$ heure à $I$ heure, à une température comprise entre $3 I^{\circ} \mathrm{C}$ et $38^{\circ} \mathrm{C}$.

Reçu pour publication le 6 avril 1957 .

\section{RÉFÉRENCES BIBLIOGRAPHIQUES}

Du Messir, Du Buisson (F.). - Ann. Zootech., facs. 3, I95-2 I2, I956.

Ito (S.), Niwa ('T.), Kudo (A.) et Mizuno (A.). - Zootech. Exp. Sta. Chiba Res. Bull., 55, I5-56, I948.

Lasley (J. F.) et Phillips (P. H.). - Missouri Exp. Sta. J., 567, 1939.

LEWIS (L. L.). - Okla Agr. Exp. Sta. Bull., 96, IgI I.

MC Kenzie (F. F.), Milier (J. C.) et Bauguess (L. C.). - Univ. Missouri Agri. Exp. Ser. Res. Bull., 279, 1938.

Mrrovayov (V.) et Halibulin (K. H.). - Prob. Zhivotn., 5, 83-9o. (A. B. A., 1, no 4), I933.

Niwa ('T.), Mrzuho (A.) et Ishikawa (S.). - Bull. Nat. Inst. Agric. Sci., $\mathrm{G}, 3,88-100$, I952.

NoI., (D. O.). - Phillip. J. Anim. Ind., 10, 247-259, I949.

Polge (C.). - Vet. Rec., 68, 62-76, I956.

RoY (A.). - Vet. Rec., 67, 330-33I, I9.55. 